\title{
On May spectral sequence and the algebraic transfer
}

\author{
By Phan Hoàng CHƠN ${ }^{*)}$ and Lê Minh HÀ $\grave{A}^{* *}$ \\ (Communicated by Kenji FukAyA, M.J.A., Oct. 12, 2010)
}

\begin{abstract}
We give a description of the dual of W. Singer's algebraic transfer in the May spectral sequence and use this description to prove new results on the image of the algebraic transfer in higher homological degrees.
\end{abstract}

Key words: Adams spectral sequence; May spectral sequence; Steenrod algebra; algebraic transfer; hit problem.

1. Introduction. Let $\mathscr{A}$ be the $\bmod 2$ Steenrod algebra $[15,18]$. The cohomology algebra, $\operatorname{Ext}_{\mathscr{A}}^{* * *}\left(\mathbf{F}_{2}, \mathbf{F}_{2}\right)$, is a central object of study in algebraic topology since it is the $E_{2}$-term of the Adams spectral sequence converging to the stable homotopy groups of the spheres [1]. However, it is notoriously difficult to compute. In fact, only quite recently has the additive structure of $\operatorname{Ext}_{\mathscr{A}}^{4, *}\left(\mathbf{F}_{2}, \mathbf{F}_{2}\right)$ been completely determined [11]. One approach to better understand the structure of this cohomology was proposed by W. Singer in [22] where he introduced an algebra homomorphism from a certain subquotient of a divided power algebra to the cohomology of the Steenrod algebra. We will call this map the algebraic transfer, because it can be considered as the $E_{2}$-level in the Adams spectral sequence of the stable transfer $B(\mathbf{Z} / 2)_{+}^{s} \rightarrow$ $S^{0}[17]$.

Let $V_{s}$ denote a $s$-dimensional $\mathbf{F}_{2}$-vector space. Its mod 2 homology is a divided algebra on $s$ generators. Let $P H_{*}\left(B V_{s}\right)$ be the subspace of $H_{*}\left(B V_{s}\right)$ consisting of all elements that are annihilated by all positive degree Steenrod squares. Let $G L_{s}=G L\left(V_{s}\right)$ be the automorphism group of $V_{s}$. It is well-known that the (right) action of the Steenrod algebra and the action of $G L_{s}$ on $H_{*}\left(B V_{s}\right)$ commute. Thus, there is an induced action of $G L_{s}$ on $P H_{*}\left(B V_{s}\right)$. For each $s \geq 0$, the rank $s$ algebraic transfer, constructed by W. Singer [22], is an $\mathbf{F}_{2^{-}}$ linear map:

2000 Mathematics Subject Classification. Primary 55R12, 55Q45, 55S10, 55T15.

*) Department of Mathematics, College of Science, Cantho University, 3/2 St, Ninh Kieu, Cantho, Vietnam.

**) Department of Mathematics-Mechanics-Informatics, Vietnam National University - Hanoi, 334 Nguyen Trai St, Thanh Xuan, Hanoi, Vietnam.

$$
\varphi_{s}: \mathbf{F}_{2} \otimes_{G L_{s}} P H_{*}\left(B V_{s}\right) \rightarrow \operatorname{Ext}_{\mathscr{A}}^{s, s+*}\left(\mathbf{F}_{2}, \mathbf{F}_{2}\right),
$$

which is known to be an isomorphism for $s \leq 3$ (this is due to Singer himself [22] for $s \leq 2$ and to Boardman [3] for $s=3$.) Moreover, the "total" transfer $\varphi=\bigoplus_{s} \varphi_{s}$ is an algebra homomorphism [22]. This shows that the algebraic transfer is highly nontrivial and should be an useful tool to study the cohomology of the Steenrod algebra. In particular, we want to know how big the image of the transfer in $\operatorname{Ext}_{\mathscr{A}}^{s, s+*}\left(\mathbf{F}_{2}, \mathbf{F}_{2}\right)$ is.

In higher ranks, W. Singer showed that $\varphi_{4}$ is an isomorphism in a range and conjectured that $\varphi_{s}$ is a monomorphism for all $s$. In [5], Bruner, Hà and Hu'ng showed that the entire family of elements $\left\{g_{i}: i \geq 1\right\}$ is not in the image of the transfer, thus refuting a question of Minami concerning the socalled new doomsday conjecture. Here we are using the standard notation of elements in the cohomology of the Steenrod algebra as was used in $[4,11,23]$.

One of the main results of this paper is the proof that all elements in the family $p_{i}$ are in the image of the rank 4 algebraic transfer. Combining the results of Hưng [8], Hà [7] and Nam [19], we obtain a complete picture of the behaviour of the rank 4 transfer. It should be noted that in [9], Hưng and Quỳnh claimed to have a proof that the family $\left\{p_{i}: i \geq 0\right\}$ is also in the image of $\varphi_{4}$, but the details have not appeared. Our work is independent from their, and our method is completely different.

Very little information is known when $s \geq 5$. At least, it is known that $\varphi_{5}$ is not an epimorphism [22]. In fact, Quỳnh [21] showed that $P h_{2}$ is not in the image of $\varphi_{5}$. We have also been able to show, [6], several non-detection results in even higher rank using the lambda algebra. For example, $h_{1} P h_{1}$ as 
well as $h_{0} P h_{2}$ are not in the image of $\varphi_{6} ; h_{1}^{2} P h_{1}$ is not in the image of $\varphi_{7}$. Often, these results are available because it is possible to compute the domain of the algebraic transfer in the given bidegree.

In this paper, we give a description of the dual of the algebraic transfer $\varphi_{s}^{*}$ in the May spectral sequence. Using this method, we recover, with much less computation, results in $[6,21]$ and [9]. Moreover, our method can also be applied, as illustrated in the case of the generator $h_{0}^{n} i, 0 \leq$ $n \leq 5$ and $h_{0}^{n} j, 0 \leq n \leq 2$, to degrees where computation of the domain of the algebraic transfer seems out of reach at the moment.

The details of this note will be published else where.

2. May spectral sequence. In this section we recall the setup for the May spectral sequence, following [13] and [14].

2.1. Associated graded algebra of the Steenrod algebra. The Steenrod algebra is a cocommutative Hopf algebra [15] whose augmentation ideal will be denoted by $\mathscr{\mathscr { A }}$. The associated augmented filtration is defined as follows:

$$
F_{p}(\mathscr{A})= \begin{cases}\mathscr{A}, & p \geq 0 \\ (\overline{\mathscr{A}})^{-p}, & p<0 .\end{cases}
$$

Let $E^{0} \mathscr{A}=\oplus_{p, q} E_{p, q}^{0} \mathscr{A}$ be the associated graded algebra. This is a bigraded algebra, where $E_{p, q}^{0} \mathscr{A}=$ $\left(F_{p} \mathscr{A} / F_{p-1} \mathscr{A}\right)_{p+q}$. According to May [14], $E^{0} \mathscr{A}$ is a primitively generated Hopf algebra on the Milnor generators $\left\{P_{j}^{i} \mid j \geq 1, i \geq 0\right\}$ (See also [15]). Its cohomology is described in the following theorem.

Theorem $2.1[14,23] . \quad H^{*}\left(E^{0} \mathscr{A}\right)$ is the homology of a complex $\mathscr{R}$, where $\mathscr{R}$ is a polynomial algebra over $\mathbf{F}_{2}$ generated by $\left\{R_{i, j} \mid i \geq 0, j \geq 1\right\}$ of degree $2^{i}\left(2^{j}-1\right)$, and its differential $\delta$ is given by

$$
\delta\left(R_{i, j}\right)=\sum_{k=1}^{j-1} R_{i, k} R_{i+k, j-k} .
$$

The coKoszul complex $\mathscr{R}$ is a quotient of the cobar complex of $E^{0} \mathscr{A}$ (see [20]), and $R_{i, j}$ is the image of $\left\{\left(P_{j}^{i}\right)^{*}\right\}$.

Remark 2.2. It is more convenient for our purposes to work with the homology version. The dual complex, denoted as $\bar{X}$ in [14], is an algebra with divided powers on the generators $P_{j}^{i}$. In fact, $\bar{X}$ is imbedded in the bar construction for $E^{0} \mathscr{A}$ (which is isomorphic to $E^{1}$-term of May spectral sequence) by sending $\gamma_{n}\left(P_{j}^{i}\right)$ to

$$
\left.\left\{P_{j}^{i}\left|P_{j}^{i}\right| \ldots \mid P_{j}^{i}\right\} \quad \text { ( } n \text { factors }\right)
$$

and the product in $\bar{X}$ corresponds to the shuffle product (see $[2$, p. 40]). Note that the image of a cycle under this imbedding is not necessary a cycle in the bar construction for $E^{0} \mathscr{A}$, so we have to add some elements if needed. This imbedding technique was succesfully exploited by Tangora [23, Chapter 5] to compute of the cohomology of the $\bmod 2$ Steenrod algebra, up to a certain range.

2.2. May spectral sequence. Let $M$ be a left $\mathscr{A}$-module of finite type, bounded below. $M$ admits a filtration, induced by the filtration of $\mathscr{A}$, given by

$$
F_{p} M=F_{p} \mathscr{A} M \text {. }
$$

It is clear that $F_{p} M=\mathscr{A} M=M$ if $p \geq 0$, and $\bigcap_{p} F_{p} M=0$.

$$
E_{p, q}^{0} M=\left(F_{p} M / F_{p-1} M\right)_{p+q} ; E^{0} M=\bigoplus_{p, q} E_{p, q}^{0} M .
$$

Then $E^{0} M$ is a bigraded $E^{0} \mathscr{A}$-module, associated to $M$.

Let $\bar{B}_{*}(M)=\bar{B}_{*}(\mathscr{A} ; M)$ be the usual bar construction with induced filtration given by

$$
F_{p} \bar{B}_{*}(M)=\sum F_{p_{1}} \overline{\mathscr{A}} \otimes \cdots \otimes F_{p_{n}} \overline{\mathscr{A}} \otimes F_{p_{0}} M,
$$

where the sum is taken over all $\left\{p_{0}, \ldots, p_{n}\right\}$ such that $n+\sum_{i=0}^{n} p_{i} \leq p$.

Theorem 2.3 [14]. Let $M$ be a $\mathscr{A}$-module of finite type, bounded below. There exists a spectral sequence converging to $H_{*}(\mathscr{A}, M)$, whose $E^{2}$-term is $E_{p, q, t}^{2} \cong H_{*}\left(E^{0} \mathscr{A}, E^{0} M\right)_{-q, q+t}$ and the differentials are $\mathbf{F}_{2}$-linear maps

$$
d^{r}: E_{p, q, t}^{r} \longrightarrow E_{p-r, q+r-1, t}^{r}
$$

3. The algebraic transfer. The stable transfer $\pi_{*}\left(B V_{s}\right)_{+} \rightarrow \pi_{*}\left(S^{0}\right)$ admits an algebraic analogue at the $E^{2}$ level of the May spectral sequence. In this section, we give an explicit description of the algebraic transfer in this $E^{2}$ level. Because of naturality, it will be clear from the construction that there is a commutative diagram

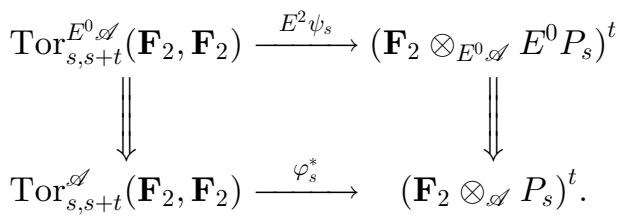


Let $\hat{P}_{1}$ be the unique $\mathscr{A}$-module extension of $H^{*}\left(\mathbf{R} P^{\infty}\right)=P_{1}=\mathbf{F}_{2}\left[x_{1}\right]$ by formally adding a generator $x_{1}^{-1}$ of degree -1 and require that $S q\left(x_{1}^{-1}\right) S q\left(x_{1}\right)=1$. Let $u: \mathscr{A} \rightarrow \hat{P}_{1}$ be the unique $\mathscr{A}$-homomorphism that sends $\theta$ to $\theta\left(x_{1}^{-1}\right)$, and put $\psi_{1}=\left.u\right|_{\mathscr{\mathscr { A }}}: \overline{\mathscr{A}} \rightarrow P_{1}$. By induction, we define

$$
\begin{gathered}
\psi_{s}: \overline{\mathscr{A}}^{\otimes s} \rightarrow P_{s}, \\
\psi_{s}\left(\left\{\theta_{s}|\ldots| \theta_{1}\right\}\right) \\
\quad=\sum_{\operatorname{deg} \theta_{s}^{\prime}>0} \theta_{s}^{\prime}\left(x_{s}^{-1}\right) \theta_{s}^{\prime \prime}\left(\psi_{s-1}\left(\left\{\theta_{s-1}|\ldots| \theta_{1}\right\}\right)\right),
\end{gathered}
$$

where we use standard notation for coproduct $\Delta(\theta)=\sum \theta^{\prime} \otimes \theta^{\prime \prime}$

It is known, from a theorem of Nam [19], that $\psi_{s}$ is a representation for the algebraic transfer on the bar construction. That is, $\psi_{s}$ induces the dual of the algebraic transfer

$\varphi_{s}^{*}: \operatorname{Tor}_{s, s+t}^{\mathscr{A}}\left(\mathbf{F}_{2}, \mathbf{F}_{2}\right) \rightarrow \operatorname{Tor}_{0, t}^{\mathscr{A}}\left(\mathbf{F}_{2}, P_{s}\right)=\left(\mathbf{F}_{2} \otimes_{\mathscr{A}} P_{s}\right)^{t}$.

We use $\psi_{s}$ to construct a chain map

$$
\tilde{\psi}_{s}: \bar{B}_{*}\left(\mathscr{A} ; \mathbf{F}_{2}\right) \rightarrow \bar{B}_{*-s}\left(\mathscr{A} ; P_{s}\right),
$$

between the bar constructions as follows. Write $\bar{B}_{n}\left(\mathbf{F}_{2}\right)=\bar{B}_{n-s}\left(\mathbf{F}_{2}\right) \otimes \overline{\mathscr{A}}^{s}$, then $\tilde{\psi}_{s}=1 \otimes \psi_{s}$, that is:

$$
\tilde{\psi}_{s}\left(\left\{\theta_{n}|\ldots| \theta_{1}\right\}\right)=\left\{\theta_{n}|\ldots| \theta_{s+1}\right\} \otimes \psi_{s}\left(\left\{\theta_{s}|\ldots| \theta_{1}\right\}\right) .
$$

Proposition 3.1. $\quad \tilde{\psi}_{s}$ is a chain homomorphism.

Our next result shows that $\tilde{\psi}_{s}$, for each $s \geq 1$, respects the May filtration.

Proposition 3.2. For each $p \leq 0, \quad s \geq 1$, there is an induced chain map:

$$
F_{p} \tilde{\psi}_{s}: F_{p} \bar{B}_{*}\left(\mathbf{F}_{2}\right) \rightarrow F_{p} \bar{B}_{*-s}\left(P_{s}\right) .
$$

As a result, there is an induced map between spectral sequences

$$
E^{r} \psi_{s}: E_{p, q, t}^{r}\left(\mathbf{F}_{2}\right) \rightarrow E_{p, q-s, t-s}^{r}\left(P_{s}\right) .
$$

In particular, we obtain

$$
\begin{gathered}
E^{2} \psi_{s}(M): \operatorname{Tor}_{s, s+*}^{E^{0} \mathscr{A}}\left(E^{0} M, \mathbf{F}_{2}\right) \\
\quad \rightarrow \operatorname{Tor}_{0, *}^{E^{0} \mathscr{A}}\left(E^{0} M, E^{0} P_{s}\right) .
\end{gathered}
$$

When $M=\mathbf{F}_{2}, E^{2} \psi_{s}\left(\mathbf{F}_{2}\right)$ is the $E^{2}$-level of the algebraic transfer in the May spectral sequence. The following is the main theorem of this section.

Theorem 3.3. The $E^{2}$-level of the dual of Singer's algebraic transfer is induced by the chain level map

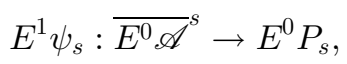

which is given inductively by

$$
\begin{aligned}
& E^{1} \psi_{s}\left(\left\{\theta_{s}|\ldots| \theta_{1}\right\}\right) \\
& \quad=\sum_{\text {deg } \theta_{s}^{\prime \prime}>0} \theta_{s}^{\prime}\left(E^{1} \psi_{s-1}\left(\left\{\theta_{s-1}|\ldots| \theta_{1}\right\}\right)\right) \theta_{s}^{\prime \prime}\left(x_{s}^{-1}\right),
\end{aligned}
$$

Because of the simple structure of $E^{0} \mathscr{A}$, it is usually quite simple to compute with $E^{1} \psi_{s}$. For example, because $P_{j}^{i}$ are primitive in $E^{0} \mathscr{A}$, we have.

Corollary 3.4. Under the chain level $E^{1} \psi_{s}$ : $\overline{E^{0} \mathscr{A}^{s}} \rightarrow E^{0} P_{s}$, the image of $\left\{P_{j_{s}}^{i_{s}}|\ldots| P_{j_{1}}^{i_{1}}\right\}$ is $x_{1}^{2^{i_{1}}\left(2^{j_{1}}-1\right)-1} \ldots x_{s}^{2^{i_{s}}\left(2^{j_{s}}-1\right)-1}$.

Theorem 3.3 and Corollary 3.4 are extremely useful to investigate the image of the algebraic transfer.

4. Two hit problems. The study of the algebraic transfer is closely related to an important problem in algebraic topology of finding a minimal basis for the set of $\mathscr{A}$-generators of the polynomial rings $P_{s}$, considered as a module over the Steenrod algebra. This is called "the hit problem" in literature [25]. A polynomial $f \in P_{s}$ is "hit" if it belongs to $\overline{\mathscr{A}} P_{s}$. There is another, related hit problem that we are going to discuss. The results in this section are crucial for applications in Section 5 and 6 .

Consider the May spectral sequence for $P_{s}$ in homological degree 0 . There are isomorphisms

$$
\begin{aligned}
E_{p,-p, t}^{2}\left(P_{s}\right) & \cong H_{0}\left(E^{0} \mathscr{A}, E^{0} P_{s}\right)_{p,-p+t} \\
& =\left(\mathbf{F}_{2} \otimes_{E^{0} \mathscr{A}}\left(E^{0} P_{s}\right)\right)_{p,-p+t},
\end{aligned}
$$

so the $E^{2}$ term concerns with the problem of determining the generators of $E^{0} P_{s}$, considered as a module over the restricted Lie algebra $E^{0} \mathscr{A}$. Determining a set of $E^{0} \mathscr{A}$-generators for $E^{0} P_{s}$ is a simpler problem, but not without difficulty, even in the rank 1 case (see [24]).

The $E^{0} \mathscr{A}$-module structure on $E^{0} P_{s}$ is related to the $\mathscr{A}$-module structure on $P_{s}$ via epimorphisms

$$
E_{p,-p, t}^{2}\left(P_{s}\right) \rightarrow E_{p,-p, t}^{\infty}\left(P_{s}\right),
$$

where in each fixed internal degree $t, E_{p,-p, t}^{\infty}\left(P_{s}\right)$ are associated graded components of $\left(\mathbf{F}_{2} \otimes \mathscr{A} P_{s}\right)^{t}$.

Given a homogeneous polynomial $f \in P_{s}$. We denote by $E^{r}(f)$ and $[f]$ the corresponding classes of $f$ in $E^{r}$ and $\mathbf{F}_{2} \otimes_{\mathscr{A}} P_{s}$ respectively. In particular, $E^{1}(f)=E^{0}(f)$ is the class of $f$ in $E^{0} P_{s}$. In order to determine $E^{r}(f)$ or $[f]$, one only needs to consider monomials in $f$ of highest filtration degree, we call this the essential part of $f$, and denote it by $\operatorname{ess}(f)$. For example, 


$$
\operatorname{ess}\left(x_{1}^{7} x_{2}^{13} x_{3}^{13}+x_{1}^{9} x_{2}^{11} x_{3}^{13}+x_{1}^{8} x_{2}^{12} x_{3}^{13}\right)=x_{1}^{7} x_{2}^{13} x_{3}^{13}
$$

because $x_{1}^{7} x_{2}^{13} x_{3}^{13}$ is in filtration -4 while the latter two monomials are in filtrations -5 and -9 respectively.

Lemma 4.1. Let $f \in P_{s}$ be a homogeneous polynomial. If $f$ is a nontrivial permanent cycle, then ess $(f)$ is non-hit in $P_{s}$.

Example 4.2. Let $m=x_{1}^{7} x_{2}^{13} x_{3}^{13} \in P_{3}$, it is not difficult to check that $m$ is nonhit in $P_{3}$. On the other hand,

$$
\begin{aligned}
m= & S q^{2}\left(x_{1}^{7} x_{2}^{11} x_{3}^{13}\right)+x_{1}^{9} x_{2}^{11} x_{3}^{13}+x_{1}^{8} x_{2}^{12} x_{3}^{13} \\
& +x_{1}^{7} x_{2}^{12} x_{3}^{14}+x_{1}^{8} x_{2}^{11} x_{3}^{14},
\end{aligned}
$$

where $x_{1}^{9} x_{2}^{11} x_{3}^{13} \in F_{-5} P_{3}$ and the last three monomials are in even smaller filtrations. Therefore

$$
E^{0}(m)=P_{1}^{1} E^{0}\left(x_{1}^{7} x_{2}^{11} x_{3}^{13}\right) \in E_{-4,37}^{0} P_{3} .
$$

So $E^{2}(m)$ is trivial.

Thus, $m$ is nonhit in $P_{s}$ then $E^{0}(m)$ is not necessary nonhit in $E^{0} P_{s}$.

Example 4.3. Consider $\quad m=x_{1} x_{2}^{2} x_{3}^{2}+$ $x_{1}^{2} x_{2} x_{3}^{2}+x_{1}^{2} x_{2}^{2} x_{3}=S q^{2}\left(x_{1} x_{2} x_{3}\right)$, so $m$ is hit in $P_{3}$. On the other hand, since $m \in F_{-2} P_{3}$ and there does not exist any element $\{\theta\} f \in F_{-1}\left(\overline{\mathscr{A}} \otimes P_{3}\right)$ such that $\theta(f)=m$ (modulo terms in $F_{p} P_{3}$ with $p<-2), E^{0}(m)$ is nonhit in $E_{-2,7}^{0} P_{3}$.

Thus, $E^{0}(m)$ is nonhit in $E^{0} P_{s}$ then $m$ is not necessary nonhit in $P_{s}$.

The following is the main result of this section.

Proposition 4.4. Let $f \in P_{s}$ be a homogeneous polynomial of filtration degree $p . f$ is a nontrivial permanent cycle if and only if $\operatorname{ess}(f)$ is non-hit in $P_{s}$ and there does not exist any non-hit polynomial $g \in F_{r} P_{s}$, with $r<p$, such that $\operatorname{ess}(f)-g$ is hit.

5. First application: a non-detection result. In this section we use the presentation in $E^{2}$-term of May spectral sequence of the dual of the algebraic transfer, constructed in section 3 , to study its image. Using this method, we are able to, not only reprove by a completely different method (with much less calculation) for results in [6,21], but also obtain the description of the image at some degrees of the algebraic transfer.

Here is our first main result.

Theorem 5.1. The following elements in the cohomology of the Steenrod algebra

(a) $h_{1} P h_{1} \in \operatorname{Ext}_{\mathscr{A}}^{6,16}\left(\mathbf{F}_{2}, \mathbf{F}_{2}\right)$;

(b) $h_{0}^{2} P h_{2} \in \operatorname{Ext}_{\mathscr{A}}^{7,18}\left(\mathbf{F}_{2}, \mathbf{F}_{2}\right)$; (c) $h_{0}^{n} i \in \operatorname{Ext}_{\mathscr{A}}^{7+n, 30+n}\left(\mathbf{F}_{2}, \mathbf{F}_{2}\right), 0 \leq n \leq 5$;

(d) $h_{0}^{n} j \in \operatorname{Ext}_{\mathscr{A}}^{7+n, 33+n}\left(\mathbf{F}_{2}, \mathbf{F}_{2}\right), 0 \leq n \leq 2$, are not detected by the algebraic transfer.

We remark that $h_{0}^{6} i=h_{0}^{3} j=0$ (see [4]).

Sketch proof. We will give the sketch of proof of (a). The proofs of other parts use similar idea.

According to Tangora [23], in $E^{1}$-term of the May spectral sequence, $h_{1} P h_{1}$ has a representation

$$
X=\left\{P_{1}^{1} \mid P_{1}^{1}\right\} *\left\{P_{2}^{0}\left|P_{2}^{0}\right| P_{2}^{0} \mid P_{2}^{0}\right\} \in E_{1} .
$$

Note that $X \in F_{-4}\left(\mathbf{F}_{2}\right)$. Here we use the same notations $P_{j}^{i}$ for elements of $\mathscr{A}$ and $E^{0} \mathscr{A}$, so $X$ can be considered as an element in $E^{1}$, being the bar construction of $E^{0} \mathscr{A}$.

Corollary 3.4 allows us to find the image of $X$ under $E^{1} \psi_{6}$ :

$$
\begin{aligned}
E^{1} \psi_{6}(X) & =x_{1} x_{2} x_{3}^{2} x_{4}^{2} x_{5}^{2} x_{6}^{2}+\text { all its permutations } \\
& =S q^{4}\left(x_{1} x_{2} x_{3} x_{4} x_{5} x_{6}\right)
\end{aligned}
$$

Therefore, $E^{1} \psi_{6}(X)$ is hit in $P_{6}$. In the bar construction, $\left(h_{1} P h_{1}\right)^{*}$ has a representation $X+x$, where $x \in F_{p} \bar{B}\left(\mathbf{F}_{2}\right)$ with $p<-4$. Thus, if $h_{1} P h_{1}$ is detected, then

$$
\psi_{6}\left(\left(h_{1} P h_{1}\right)^{*}\right)=E^{1} \psi_{6}(X)+y,
$$

where $y \in F_{p} P_{6}$ with $p<-4$, is nonhit in $P_{6}$. On the other hand, it can be verified by direct computation that there is only one possible polynomial: $x_{1}^{4} x_{2}^{4} x_{3}^{2} x_{4}^{0} x_{5}^{0} x_{6}^{0}$ (or its permutations). But it is clearly hit in $P_{6}$ as well.

It should be noted that the dimension of the above elements go far beyond the current computational knowledge of the hit problem.

Corollary $5.2[21,22] . \quad P h_{1} \in \operatorname{Ext}_{\mathscr{A}}^{5,14}\left(\mathbf{F}_{2}, \mathbf{F}_{2}\right)$ and $\mathrm{Ph}_{2} \in \operatorname{Ext}_{\mathscr{A}}^{5,16}\left(\mathbf{F}_{2}, \mathbf{F}_{2}\right)$ are not in the image of the algebraic transfer.

That these elements are not detected are known, they are due to Singer [22] and Quỳnh [21] respectively. Our proof is much less computational.

6. Second application: $p_{0}$ is in the image of the transfer. In this sections, we show that our method can also be used to detect elements in the image of the algebraic transfer. This fact completes the proof of a conjecture in [8], which provides a complete picture of the fourth algebraic transfer.

The following is our second main result.

Theorem 6.1. The element $p_{0} \in \operatorname{Ext}_{\mathscr{A}}^{4,37}\left(\mathbf{F}_{2}\right.$, $\left.\mathbf{F}_{2}\right)$ is in the image of the fourth algebraic transfer. 
This result is announced in [9], but the details have not appeared.

Since the squaring operation $S q^{0}$, defined by Kameko [10], acting on the domain of the algebraic transfer commutes with the classical $S q^{0}$ on $\operatorname{Ext}_{\mathscr{A}}^{*, *}\left(\mathbf{F}_{2}, \mathbf{F}_{2}\right)$ [12] through the algebraic transfer [16], we obtain following result.

Corollary 6.2. Every element in the family $p_{i} \in \operatorname{Ext}_{\mathscr{A}}^{4,37 \cdot 2^{i}}\left(\mathbf{F}_{2}, \mathbf{F}_{2}\right), i \geq 0$, is in the image of the algebraic transfer.

Sketch proof of Theorem 6.1. According to Tangora, $p_{0}$ is represented by $R_{0,1} R_{3,1} R_{1,3}^{2}$, so its dual $p_{0}^{*}$ is represented in $E^{1}$-term of May spectral sequence by

$\bar{p}_{0}=\left\{P_{1}^{3}\right\} *\left\{P_{1}^{0}\right\} *\left\{P_{3}^{1} \mid P_{3}^{1}\right\}+\left\{P_{1}^{3} \mid P_{1}^{3}\right\} *\left\{P_{2}^{1}\right\} *\left\{P_{4}^{0}\right\}$.

Under $E^{1} \psi_{4}$, this element is sent to (see Corollary 3.4)

$$
\begin{aligned}
\tilde{p}_{0}= & x_{1}^{0} x_{2}^{7} x_{3}^{13} x_{4}^{13}+x_{1}^{7} x_{2}^{7} x_{3}^{5} x_{4}^{14} \\
& + \text { all their permutations. }
\end{aligned}
$$

Using Example 4.2 and the fact that

$$
E^{0}\left(x_{1}^{7} x_{2}^{7} x_{3}^{5} x_{4}^{14}\right)=P_{1}^{1} E^{0}\left(x_{1}^{7} x_{2}^{7} x_{3}^{3} x_{4}^{14}\right),
$$

we see that $E^{0}\left(\tilde{p}_{0}\right)$ is hit in $E^{0} P_{4}$. Therefore, $E^{0}\left(\tilde{p}_{0}\right)$ does not survive to $E_{-4,4,33}^{\infty}\left(P_{4}\right)$.

By direct calculation, we see that, in the bar construction, $p_{0}^{*}=\bar{p}_{0}+x+y$, where $y \in F_{-6} \bar{B}\left(\mathbf{F}_{2}\right)$ and

$$
x=\left\{P_{1}^{2} \mid P_{1}^{2}\right\} *\left\{P_{2}^{1}\right\} *\left\{P_{4}^{0}\right\} .
$$

So that,

$$
\begin{aligned}
\psi_{4}\left(p_{0}^{*}\right) & =\psi_{4}\left(\bar{p}_{0}+x+y\right) \\
& =X+X(12)+X(132)+X(1432)+Y,
\end{aligned}
$$

where $(12),(132),(1432)$ are elements of the symmetric group $S_{4}$, their action permutes variables of $P_{4}$;

$$
\begin{aligned}
X= & x_{1}^{0} x_{2}^{7} x_{3}^{13} x_{4}^{13}+x_{1}^{0} x_{2}^{13} x_{3}^{7} x_{4}^{13}+x_{1}^{0} x_{2}^{13} x_{3}^{13} x_{4}^{7} \\
& +x_{1}^{0} x_{2}^{13} x_{3}^{17} x_{4}^{3}+x_{1}^{0} x_{2}^{17} x_{3}^{13} x_{4}^{3}+x_{1}^{0} x_{2}^{17} x_{3}^{3} x_{4}^{13} \\
Y= & (7,7) *(5) *(14)+(16,5,7) *(5) \\
& +(18,3,7) *(5)+(20,1,7) *(5) \\
& +(11,3,14) *(5)+(11,3) *(5) *(14) \\
& +(5,2) *(13,13)+(17,1,2) *(13) \\
& +(14,9,3,7)+(9,14,3,7)+(9,3,14,7) \\
& +(7,14,3,9)+(7,9,3,14)+(14,7,3,9) \\
& +(9,3,7,14)+(9,7,3,14)+(20,1,5,7) \\
& +(16,9,1,7)+(9,16,1,7)+(5,16,9,3) \\
& +(9,5,16,3)+(18,3,9,3)+(9,3,18,3) \\
& +(9,5,14,5)+(9,5,5,14)+(5,9,5,14) .
\end{aligned}
$$

Here we use $(a, b, c, d)$ to denote the monomial $x_{1}^{a} x_{2}^{b} x_{3}^{c} x_{4}^{d}$, and use $*$ to denote all permutations that is similar to shuffle product.

Since $X$ is hit in $P_{4}$, so are $X(12), X(132)$ and $X(1432)$.

By direct inspection, we show that $E^{0}(Y)=$ $E^{0}((3,5) *(11) *(14))$ is a nontrivial permanent cycle. According to Proposition $4.4, \psi_{4}\left(p_{0}^{*}\right)$ is nonhit in $P_{4}$. Thus, $p_{0}$ is in the image of fourth algebraic transfer.

Acknowledgments. We would like to thank Prof. Bob Bruner for his help and encouragement. The first author would like to thank Profs. J. Peter May and Wen-Hsiung Lin for their helpful answers to his questions.

This work is partially supported by the NAFOSTED grant No. 101.01.51.09.

\section{References}

[ 1 ] J. F. Adams, On the structure and applications of the Steenrod algebra, Comment. Math. Helv. 32 (1958), 180-214.

[ 2 ] J. F. Adams, On the non-existence of elements of Hopf invariant one, Ann. of Math. (2) 72 (1960), 20-104.

[ 3 ] J. M. Boardman, Modular representations on the homology of powers of real projective space, in Algebraic topology (Oaxtepec, 1991), 49-70, Contemp. Math., 146, Amer. Math. Soc., Providence, RI.

[ 4 ] R. R. Bruner, The cohomology of the mod 2 Steenrod algebra: A computer calculation, WSU Research Report 37 (1997).

[ 5 ] R. R. Bruner, L. M. Hà and N. H. V. Hưng, On the behavior of the algebraic transfer, Trans. Amer. Math. Soc. 357 (2005), no. 2, 473-487.

[6 ] P. H. Chơn and L. M. Hà, Lambda algebra and the Singer transfer. (Preprint).

[ 7 ] L. M. Hà, Sub-Hopf algebras of the Steenrod algebra and the Singer transfer, in Proceedings of the School and Conference in Algebraic Topology, 81-105, Geom. Topol. Monogr., 11 (2007), 81-105.

[ 8 ] N. H. V. Hưng, The cohomology of the Steenrod algebra and representations of the general linear groups, Trans. Amer. Math. Soc. 357 (2005), no. 10, 4065-4089.

[ 9 ] N. H. V. Hưng and V. T. N. Quỳnh, The image of Singer's fourth transfer, C. R. Math. Acad. Sci. Paris 347 (2009), no. 23-24, 1415-1418.

[10] M. Kameko, Products of projective spaces as Steenrod modules, ProQuest LLC, Ann Arbor, MI, 1990

[11 ] W.-H. Lin, $\operatorname{Ext}_{\mathscr{A}}^{4, *}(\mathbf{Z} / 2, \mathbf{Z} / 2)$ and $\operatorname{Ext}_{\mathscr{A}}^{5, *}(\mathbf{Z} / 2, \mathbf{Z} / 2)$, Topology Appl. 155 (2008), no. 5, 459-496.

[ 12 ] A. Liulevicius, The factorization of cyclic reduced powers by secondary cohomology operations, 
Mem. Amer. Math. Soc. No. 42 (1962), 112 pp.

[13] J. P. May, The cohomology of restricted Lie algebras and of Hopf algebras: Application to the Steenrod Algebra, ProQuest LLC, Ann Arbor, MI, 1964.

[14] J. P. May, The cohomology of restricted Lie algebras and -of Hopf algebras, J. Algebra 3 (1966), 123-146.

[ 15 ] J. Milnor, The Steenrod algebra and its dual, Ann. of Math. (2) 67 (1958), 150-171.

[ 16 ] N. Minami, The iterated transfer analogue of the new doomsday conjecture, Trans. Amer. Math. Soc. 351 (1999), no. 6, 2325-2351.

[17 ] S. A. Mitchell, Splitting $B(\mathbf{Z} / p)^{n}$ and $B T^{n}$ via modular representation theory, Math. Z. 189 (1985), no. 1, 1-9.

[18 ] D. B. A. Epstein, Cohomology operations: Lectures by N. E. Steenrod, Ann. Of Math. Stud., 50, Princeton Univ. Press, Princeton, NJ, 1962.

[19 ] T. N. Nam, Transfert algébrique et action du groupe linéaire sur les puissances divisées modulo 2, Ann. Inst. Fourier (Grenoble) $\mathbf{5 8}$ (2008), no. 5, 1785-1837.

[ 20 ] S. B. Priddy, Koszul resolutions, Trans. Amer. Math. Soc. 152 (1970), 39-60.

[ 21 ] V. T. N. Quỳnh, On behavior of the fifth algebraic transfer, in Proceedings of the School and Conference in Algebraic Topology, Geom. Topol. Monogr., Coventry, 11, 2007, 309-326.

[ 22 ] W. M. Singer, The transfer in homological algebra, Math. Z. 202 (1989), no. 4, 493-523.

[23] M. C. Tangora, On the cohomology of the Steenrod algebra, Math. Z. 116 (1970), 18-64.

[ 24 ] R. Vakil, On the Steenrod length of real projective spaces: finding longest chains in certain directed graphs, Discrete Math. 204 (1999), no. 1-3, 415-425.

[25] R. M. W. Wood, Problems in the Steenrod algebra, Bull. London Math. Soc. 30 (1998), no. $5,449-517$. 